\title{
Diagnóstico preliminar de la calidad del aire y del agua en centros de mayor poblamiento humano de la región del Chocó Biogeográfico colombiano
}

\section{Preliminary diagnosis of the air and water quality in main town of the region of Colombian Choco Biogeographic}

\author{
Yenecith Torres ${ }^{1}$, Tatiana López ${ }^{2}$, Fredy Carabali ${ }^{3}$
}

\section{RESUMEN}

Durante el segundo semestre de 2007 se realizaron recorridos en la región del Chocó Biogeográfico Colombiano que permitieran recolectarinformación primaria y secundaria con el propósito de establecer las condiciones de la calidad del aire y el agua. Los resultados de investigaciones compiladas y recorridos realizados en Apartadó, Turbo, Quibdó, Buenaventura, Guapi y Tumaco permitieron establecer como principales contaminantes hídricos los vertimientos de aguas domésticas, metales pesados, hidrocarburos y lixiviados generados por la inadecuada disposición de residuos sólidos. En cuanto a calidad del aire las principales fuentes de emisión son móviles, las emisiones atmosféricas de mayor concentración son monóxido de carbono (CO) e hidrocarburos (HC). El análisis sobre la incidencia de enfermedades asociadas con la calidad agua y aire manifiesta un posible detrimento de la salud de los pobladores debido en gran parte a escasos procesos de control, mitigación y compensación en los centros poblados de estudio.

Palabras clave: Calidad del aire; Calidad del agua; Contaminantes hídricos; Emisiones atmosféricas; Chocó biogeográfico.

\begin{abstract}
During the second semester of 2007, we realized in the region of the Chocó Biogeográfico Colombiano that were allowing collecting primary and secondary information with the intention of establishing the conditions of the quality of the air and water. The results of research compiled in Apartadó, Turbo, Quibdó, Buenaventura, Guapi and Tumaco, can be established as a major water pollutant discharges of domesticwater, heavy metals, hydrocarbons and leachate generated by the improper disposal of solid waste. For air quality the main emission sources are mobile where that the largest concentrations of emissions are carbon monoxide (CO) and Hydrocarbons (HC). The analysis on the incident of diseases associated with the quality waters down and air demonstrates a possible detriment of the health of the settlers owed largely to scanty processes of control, mitigation and compensation in the population centers of study.
\end{abstract}

Keywords: Air quality; Water quality; Water pollutants; Atmospheric emissions; Chocó Biogeographic.
1. Investigadora, Instituto de Investigaciones Ambientales del Pacífico. Coordinadora del proyecto. e-mail: yene8025@yahoo.com

2. Contratista, Instituto de Investigaciones Ambientales del Pacífico. Investigadora del proyecto, Pacífico sur.

e-mail: taticologa@gmail.com

3. Contratista, Instituto de Investigaciones Ambientales del Pacífico. Coordinador SIG del proyecto.

Recibido: Septiembre 28, 2007

Aceptado: Enero 15, 2008 


\section{Bioetnia Volumen 5 No 1 (enero-junio), 2008}

\section{INTRODUCCIÓN}

La calidad ambiental es un factor determinante para el desarrollo de las comunidades de flora y fauna y una prioridad para la salud humana. El deterioro de sus componentes genera repercusiones indeseables sobre la economía de una nación y el bienestar de la población. Los avances científicos e investigaciones realizadas mediante vigilancia ambiental (Invemar, CVC, Corpourabá, Codechocó, Corponariño 2006) y epidemiológica (Muñoz, Quiróz y Paz; 2006), demuestran relaciones de respuestas cada vez más rápidas entre el estado de calidad del ambiente y calidad de vida, incluyendo el equilibrio entre sus variables. Las afecciones que se presentan de manera aguda y crónica sobre los seres humanos y ecosistemas expuestos a constante contaminación, tienen un costo social que aumenta constantemente en las regiones menos favorecidas, como es el caso del Chocó Biogeográfico Colombiano, donde, el desarrollo tecnológico centrado en la explotación primaria, ha provocado la contaminación y disminución de su calidad ambiental, afectando las bases del ecosistema.

Por lo anterior, el presente estudio prioriza los efectos generados por la interacción de dinámicas socioculturales y económicas sobre los recursos aire y agua, e identifica la gestión de corporaciones autónomas regionales, universidades e instituciones ambientales, alrededor de la medición de diferentes contaminantes, lo cual permite identificar vacíos de información y la manera de abordar la investigación en la región del Chocó Biogeográfico Colombiano.

\section{MATERIALES Y MÉTODOS}

Área de estudio. El Chocó Biogeográfico es una región neotropical, localizada desde el oeste de la costa Caribe y la provincia del Darién al oeste de Panamá, hasta la costa pacífica del oeste de Colombia y el noroeste de Ecuador y extremo norte del Perú; incluye además la región de Urabá y el valle medio del río Magdalena y sus afluentes Cauca-Nechí y San Jorge. En Colombia esta región se conoce como la región del andán Pacífico donde se incluye el departamento del Chocó, parte de los departamentos de Antioquia, Risaralda, Valle del Cauca, Cauca y Nariño. El presente estudio se desarrolló en los municipios de Apartadó, Turbo, Quibdó, Buenaventura, Guapi y Tumaco (Figura 1).

Apartadó: Municipio ubicado al norte del departamento de Antioquia, con una población de 134.572 habitantes; es el poblado con mayor actividad agrícola de la región. La fuente de empleo principal es la recolección, preparación y exportación de banano.

Turbo: Localizado en la zona bananera, al norte del departamento de Antioquia. Cuenta con 122.780 habitantes.

Quibdó: Quibdó es la capital del departamento del Chocó, situado hacia el nor-occidente del país. La ciudad se encuentra a orillas del río Atrato, uno de los principales afluentes de Colombia y una de las zonas con más alta pluviosidad del mundo. Su población es de 105.172 habitantes.

Buenaventura: Se encuentra localizada en el departamento del Valle del Cauca, en la bahía interior del Océano Pacífico. Cuenta con un total de 307.730 habitantes. Es el puerto marítimo con mayor movilización (7'645.000 toneladas) de productos de importación y exportación del Océano Pacífico (MinTransporte, 2005).

Guapi: Se encuentra ubicado al sur occidente del departamento del Cauca, bordeando la vertiente del Pacífico colombiano. Su población se estima en 30.000 habitantes.

Tumaco: Se localiza en el sur occidente del departamento de Nariño. Es el segundo puerto marítimo en la costa pacífica. Tumaco es el centro poblado con mayor actividad industrial en el pacífico colombiano, en cuanto a transformación de materia prima.

Métodos. El diagnóstico se basó principalmente en la recopilación de información secundaria, de diferentes Corporaciones Autónomas Regionales (CARs), universidades públicas y privadas, alcaldias municipales (planeación, saneamiento básico) y hospitales municipales; en adición se contempló información proporcionada en entrevistas a personas de amplia trayectoria en los diferentes temas y habitantes de zonas aledañas a sitios con alto grado de contaminación. Se consultaron registros de enfermedades diarreicas agudas (EDA) e infecciones respiratorias agudas (IRA) en los hospitales de Apartadó, Turbo, Quibdó y Buenaventura, información que permitió calcular el número de habitantes afectados posiblemente por agua y aire contaminado.

De manera complementaria, se elaboró cartografia para identificar los puntos de muestreo del agua y su calidad, basados en los contaminantes 
de mayor importancia. Para la generación de isolíneas de $\mathrm{pH}$, temperatura, oxígeno disuelto y conductividad, se recopiló información ya capturada por la CVC, Corpourabá, Codechocó e INVEMAR. La información recolectada se unificó a formato de tablas (*.dbf); estos datos se analizaron con el método de interpolación de Kriging usando el software Surfer (Golden software, 2002). Finalmente, con la ayuda del software de SIG ArcView 3.2 (ESRI, 2000), se realizó la visualización de los resultados de interpolación, para las variables del recurso hídrico, temperatura, oxígeno disuelto, $\mathrm{pH}$ y conductividad.

\section{RESULTADOSY DISCUSIÓN}

En total se colectaron 41 documentos de estudios realizados con calidad del aire y el agua en centros de mayor población humana de la región del Chocó Biogeográfico Colombiano.

Resultados generales por centros poblados. La ejecución de investigaciones está asociada con el desarrollo económico de los centros poblados y con la capacidad técnica de las corporaciones autónomas regionales. La información resulta ser mayor para las

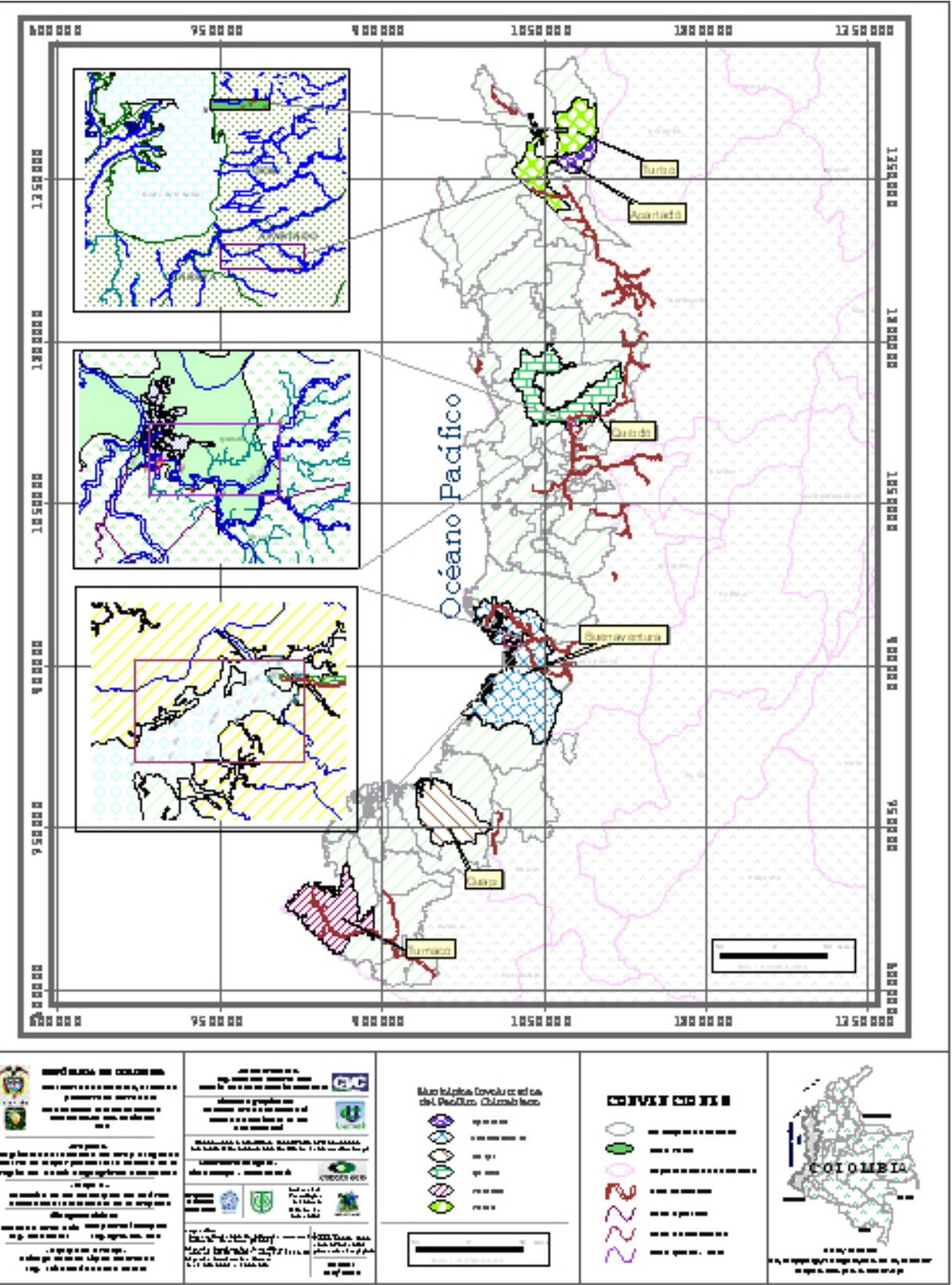

Figura 1. Ubicación del área de estudio: centros de mayor poblamiento humano de la región del

Chocó Biogeográfico Colombiano

Fuente: Corpourabá, CVC, Codechocó, IIAP, 2007 ciudades de Turbo y Buenaventura lo cual se soporta en la existencia de universidades y en la mayor capacidad técnica de las CARs (Gráfico 1).

Resultados generales por componente. La información generada en los centros de estudio, evidencia la disparidad de datos entre los componentes agua y aire. El componente calidad del agua contiene $78 \%$ de los documentos compila- dos, seguido del mixto (componente calidad del agua y el aire) con 17\%, mientras las investigaciones relacionadas con el componente atmosférico representan sólo 5\%. En el componente calidad del agua, se reconocen los esfuerzos del proyecto REDCAM (INVEMAR y CARs), como una de las principales iniciativas de monitoreo permanente que genera datos sobre variaciones de 


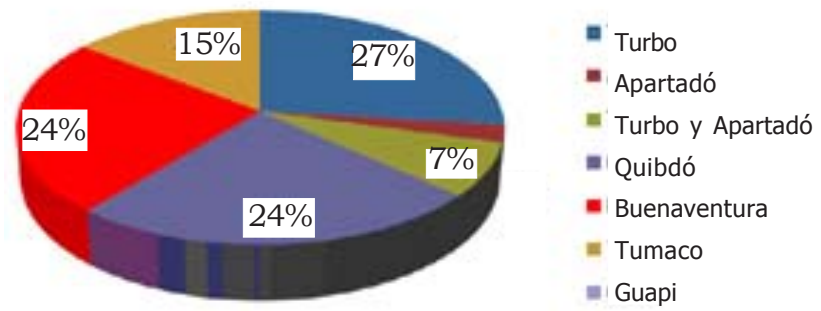

Gráfico 1. Porcentaje de información en calidad del aire y el agua generada por los centros de mayor poblamiento humano del Chocó Biogeográfico.

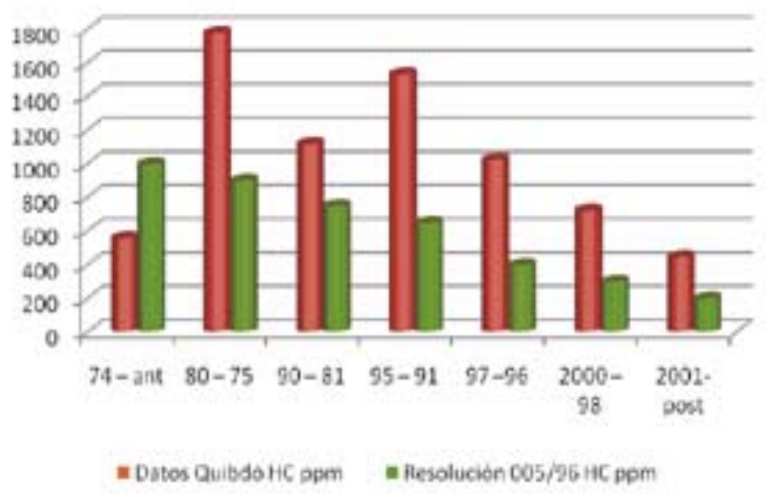

Gráfico 2. Promedio de emisiones de transporte de servicio público en Quibdó en comparación con la Resolución 005/96.

Fuente: Datos suministrados por Andrade y Córdoba, 2005

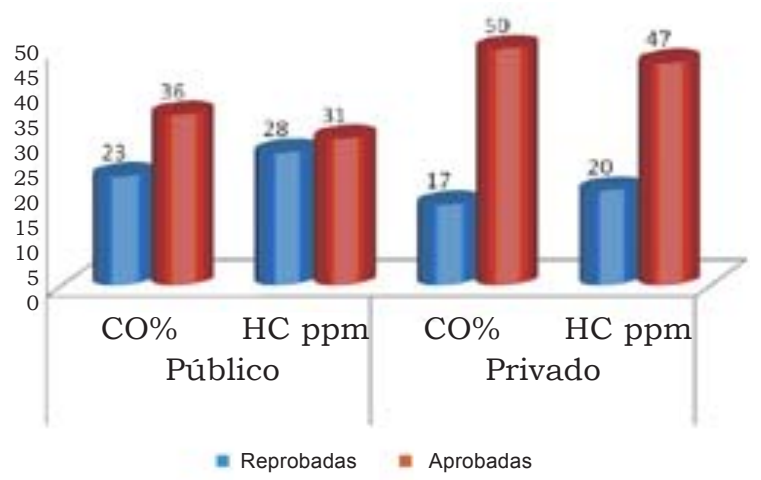

Gráfico 3. Número de motocicletas aprobadas y reprobadas por emisiones de $\mathrm{CO}$ y $\mathrm{HC}$ en el municipio de Quibdó.

Fuente: Cuesta y Reales, 2007

parámetros fisicoquímicos de aguas marinas y continentales en la región.

Calidad del aire. Las concentraciones máximas del fungicida cloratonil medidos en la zona bananera, se informan para el municipio de

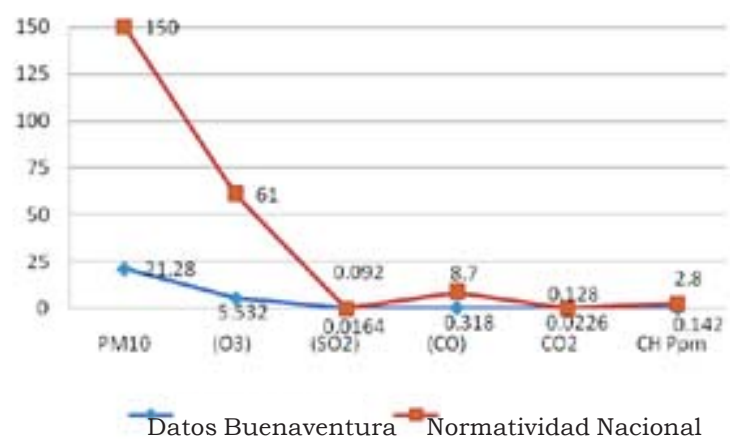

Gráfico 4. Promedio de gases y partículas contaminantes medidos en Buenaventura, comparados con la norma nacional.

Fuente: CVC, 2000

Apartadó (AUGURAy Corpouraba, 2000). En Quibdó las únicas fuentes de contaminación atmosférica son las que se refieren a focos móviles como son emisiones por vehículos de servicio público (buses y motocicletas). De acuerdo con Andrade y Córdoba (2004), sólo los autos modelo 1974 no sobrepasan los niveles de HC permisibles por la resolución 005/96 (Gráfico 2). En relación con los niveles de CO los modelos 1974, 1990-1981 y 1995-1991 sobrepasan los niveles permisibles por la resolución. El monitoreo de emisiones de gases por motocicletas (Cuesta y Reales, 2007) muestra que $50.8 \%$ sobrepasan la media de concentración de gases por año (Gráfico 3). Lo anterior se debe a que sólo 6.3\% del total de vehículos utiliza como combustible gasolina extra; en adición, gran parte de los vehículos no reciben mantenimiento oportuno, lo cual aumenta el riesgo de emisión de estos gases.

En Buenaventura los niveles de PM10, monóxido y dióxido de nitrógeno evidencian la influencia del parque automotor, sin sobrepasar los niveles de la norma nacional aún en los meses de mayor emisión para cada parámetro (Gráfico 4). Por otra parte, el flujo vehicular genera $80 \%$ del ruido urbano, seguido por las actividades comerciales y comunitarias que generan $10 \%$ cada una (Rosero, 2000); estos niveles sobrepasan los limites del Decreto 948/95 en un promedio diario equivalente a $16.2 \%$ (vehicular) y $15 \%$ (comercial).

Los niveles de monóxido de carbono, monóxido de azufre y partículas móviles evaluados en el aeropuerto y las empresas de palma aceitera en Tumaco, se encuentran por debajo de los niveles 
mínimos de emisión de la norma nacional colombiana (Tabla 1) (Análisis Ambiental, 2003, 2005, 2006, 2007; UAEAC, 2006).

Efectos de la contaminación del aire sobre la salud humana. Los datos de morbilidad suministrados por los hospitales de Apartadó, Turbo, Quibdó y Buenaventura en relación con los casos de IRA informados desde enero a octubre de 2007 en niños menores de cinco (5) años, muestran que en la región de 5.285 casos, 2.399 (45\%) son en Buenaventura, seguida de Apartadó con 1.743 (33\%). Quibdó y Turbo con $742(14 \%)$ y 401 (8\%), informan los valores más bajos en este tipo de consultas. No obstante, la mortalidad por este tipo de infecciones es baja teniendo en cuenta la incidencia de afectación en la población, pues el informe del hospital de Buenaventura registró dos muertes por IRA para el año 2007.

Calidad del agua. En los municipios de Turbo y Apartadó se han detectado trazas de aldrín, endrín y lindano, sobre todo en cuerpos de agua dulce. Las mayores concentraciones de Lindano se registran para el río Turbo (Gráfico 5); en este río las concentraciones más altas de plaguicidas, corresponden a los isómeros de hexaclorociclohexano (Gráfico 6), siendo mayores para CHC (Roqueme y Blanco, 2005). En el río Apartadó se registró la presencia de clorotalonil con un nivel de concentración de $25.10 \mathrm{~g} / 1 \mathrm{EPA}$ (Corpourabá-Augura, 2000), superando la norma establecida por la EPA. En relación con los parámetros fisicoquímicos y microbiológicos medidos en los ríos Apartadó y Turbo, en general los valores de temperatura, $\mathrm{pH}$ y oxígeno disuelto, se encuentran dentro de los rangos establecidos por la normatividad nacional colombiana, mientras

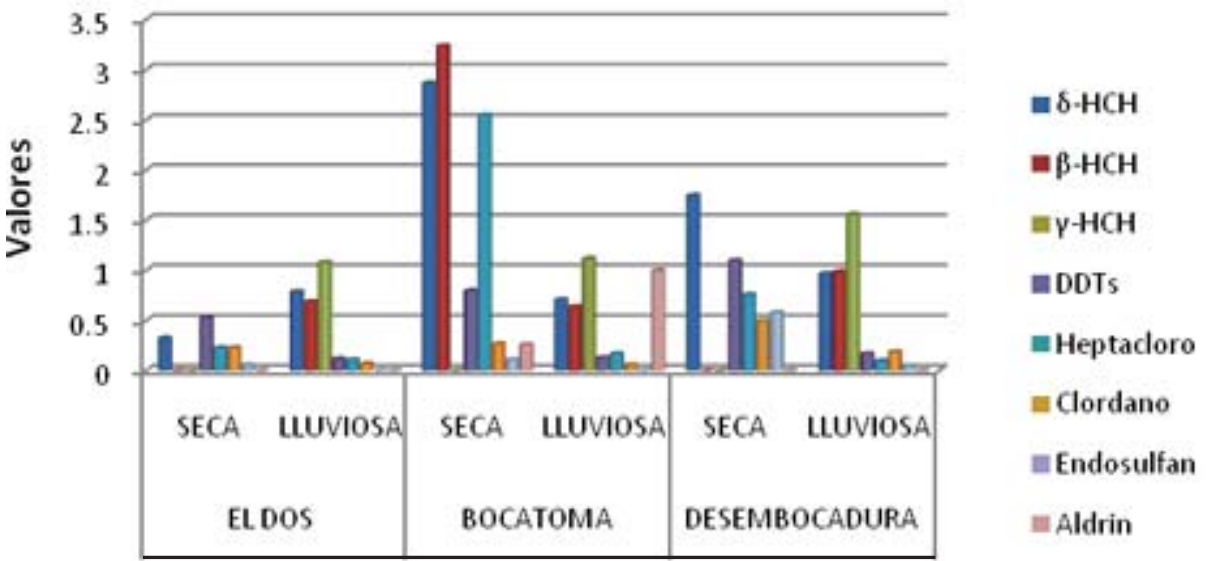

Estaciones de Estudio

Gráfico 5. Concentración de plaguicidas organoclorados en sedimentos del río Turbo durante la época seca y lluviosa

Fuente: Modificado de Roqueme y Blanco, 2005

$=\operatorname{ALD}(\mathbf{n g} / \mathbf{l})=\operatorname{END}(\mathbf{n g} / \mathbf{l})=\operatorname{LND}(\mathbf{n g} / \mathbf{l})=\operatorname{DDD}(\mathbf{n g} / \mathbf{l})=\operatorname{DDT}(\mathbf{n g} / \mathbf{l})$

Gráfico 6. Concentración de plaguicidas organoclorados en ecosistemas marinos y dulceacuícolas

Fuente: Datos suministrados por Corpourabá, 2004

Tabla 1

Niveles de parámetros contaminantes medidos $(\mathrm{kg} / \mathrm{h})$ en empresas extractoras de palma de aceite en Tumaco

\begin{tabular}{|c|c|c|}
\hline Empresa & Sox & Nox \\
\hline
\end{tabular}

Fuentes fijas

$\begin{array}{llll}\text { Santa Elena } & 0,01 & 0,12 & 1,07 \\ \text { Palmapac } & 0,06 & 0,02 & 1,95 \\ \text { Astorga } & 0,12 & 0,06 & 1,54 \\ \text { Araki } & 0,01 & 0,06 & 0,59\end{array}$

Fuentes móviles

$\begin{array}{llll}\text { Aeropuerto } & 0,005 & 0,0515 & 0,48\end{array}$


Bioetnia Volumen 5 No 1 (enero-junio), 2008

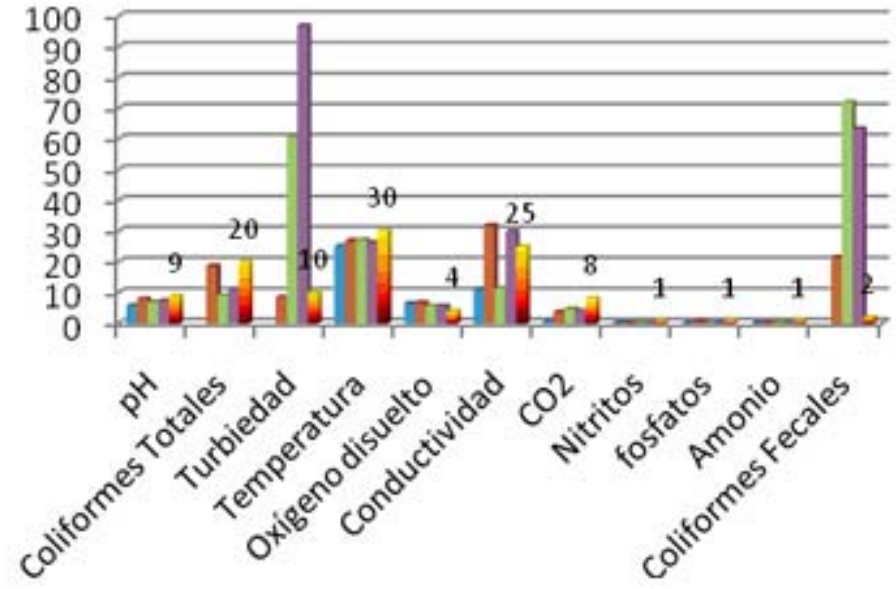

Gráfico 7. Variables fisicoquímicas monitoreadas en las quebradas La Francisca, La Paloma, La Aurora, Calle Quibdó (Río Atrato) en relación con los límites establecidos por la Normatividad Nacional.

* Coliformes expresados en miles.

Fuente: Datos tomados de Bejarano y Palacios; Torres y Castro; Milan y Beltrán, 2004; Marín y Moreno, 2006

que variables como turbiedad, coliformes totales y fecales superan los valores permitidos para la preservación de flora y fauna acuática. La distribución y abundancia de macroinvertebrados en aguas marinas y costeras del golfo de Urabá, está relacionada sobre todo con las fluctuaciones de los niveles de salinidad (García y Correa, 2006; Gaviria, 2006) mientras que las concentraciones de nitrógeno inorgánico disuelto aumentan en zonas cercanas a plantaciones de banano, haciendo de ésta una de las zonas costeras del país más afectadas por dispersión de hidrocarburos disueltos (Invemar y CARs, 2002).

En Quibdó, estudios realizados en el río Atrato y afluentes como Cabí, La Paloma, La Aurora y La Francisca, muestran que debido al vertimiento de residuos líquidos y sólidos y al arrastre de sedimentos, las variables turbiedad y coliformes fecales superan los limites establecidos por la norma nacional de calidad del agua (Álvarez y Cuesta, 2005; Caicedo y Mena, 2005; Palacios y Pino, 2005; Marín y Moreno, 2005). Si bien, los niveles de oxígeno disuelto en algunos afluentes del río Atrato se encuentran por encima del valor establecido por la normatividad, no son perjudiciales para el desarrollo de la vida acuática (Gráfico 7). Evaluaciones sobre la calidad del agua utilizando macroinvertebrados acuáticos dulceacuícolas como indicadores, demuestran que algunos afluentes de la cuenca del río Atrato se encuentran moderadamente contaminados (Castro y Torres, 2004; Palacios y Romaña, 2004; Bejarano y Palacios, 2004).

En Buenaventura, los promedios de parámetros fisicoquímicos básicos medidos en los ríos San Juan, Raposo, Potedo, Anchicayá y Dagua, muestran que las variables se encuentran entre los límites permisibles por la normatividad, a excepción de la turbiedad, la cual supera ampliamente los rangos establecidos (Gráfico 8). Aunque se observa contaminación por hidrocarburos, plomo y mercurio, las concentraciones no superan las establecidas por la EPA (Lozano, 1998; Ortega, 1996). El plomo y cobre arrojado por empresas mineras en el río Dagua, se acumulam en valores altamente significativos en las macroalgas Bostrychia callipteray Rhizoclonium riparium sobrepasando los limites establecidos por la normatividad nacional (Lozano, 2006). El otro factor de vertimiento determinante en la contaminación del agua en Buenaventura, se refiere a las aguas residuales provenientes de la transformación industrial pesquera, donde se generan vertimientos de demanda biológica de oxígeno (DBO) y demanda química de oxígeno (DQO) de $3.051 \mathrm{mgO}_{2} / 1$ y $3.850 \mathrm{mgO}_{2} / 1$ respectivamente, valores que sobrepasan la norma de calidad del agua (Hinestroza, 2002). La población macrobentónica de la bahía de Buenaventura presenta mayor abundancia en áreas de descargas orgánicas debido al aumento de sedimentos, lo cual favorece la riqueza de especies encontrando variaciones de riqueza de 21 especies (Tovar, 1995).

De acuerdo con los análisis ambientales de las empresas aceiteras en Tumaco, las emisiones de contaminantes presentan valores de efluentes bastante elevados. No obstante las empresas cumplen los estándares de la norma debido a que los valores de los efluentes presentan los porcentajes de remoción contemplados en el Artículo 72 del Decreto 1594/84 con una eficiencia hasta de 


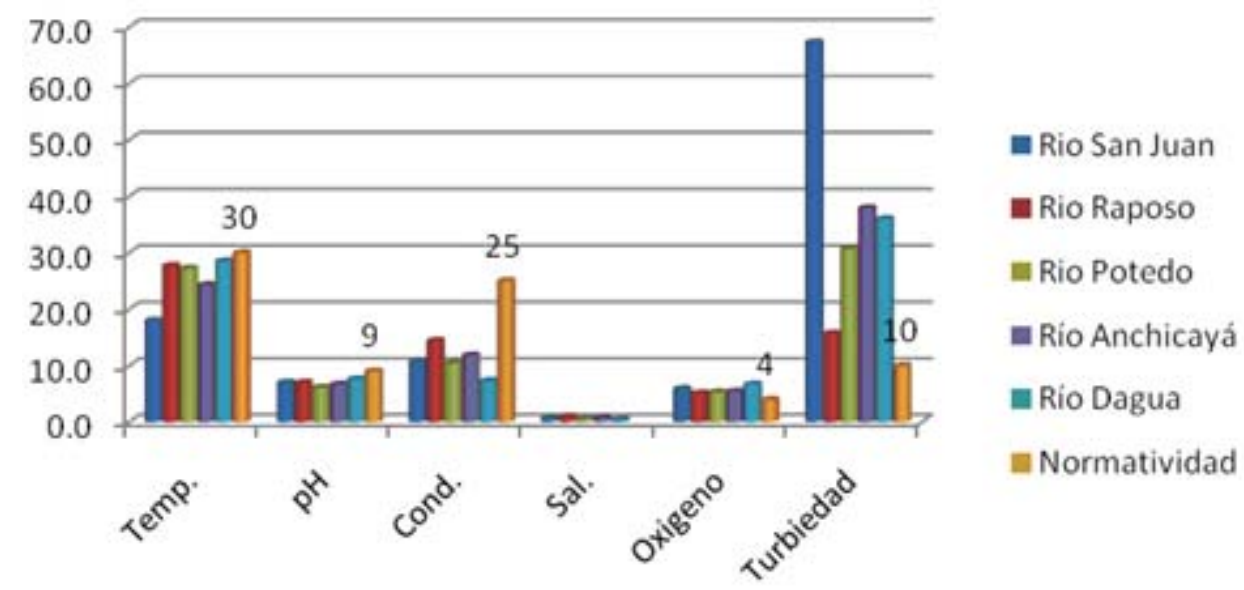

Gráfico 8. Variables fisicoquímicas monitoreadas en los ríos principales aledaños a la ciudad de Buenaventura con relación a los límites establecidos por la Normatividad.

Fuente: Datos suministrados por CVC, 2006

99\% (Tabla 2).

Una de las principales causas de contaminación hídrica se debe a vertimientos que no están sujetos a sistemas de tratamiento, sobre todo a nivel doméstico; en total $87.211 \mathrm{~m}^{3} /$ día de aguas servidas urbanas son vertidas en zonas costeras a través de las cuales se aportan 2.97 ton/día de nitrógeno total, 0.59 ton/día de fosfatos, 10.7 ton/ día de sólidos suspendidos y 6.35 NMP/día de coliformes fecales (Invemar, 2004).

Efectos de la contaminación del aire sobre la salud humana. Los datos de morbilidad de urgencias suministrados por los hospitales de Apartadó, Turbo, Quibdó y Buenaventura en relación con los casos de EDA informados entre enero y octubre de 2007 en niños menores de cinco (5) años, muestran que de 4.349 casos, 1.750 (40\%) se registran en Turbo, seguido de Buenaventura con 1.435 casos. Apartadó y Quibdó con 746 y 418 consultas por urgencia respectivamente, repor$\tan 37 \%$ de los casos de EDA.

\section{CONCLUSIONES}

Los porcentajes de emisiones al aire aunque no representan una amenaza, no indican la calidad atmosférica en las ciudades de estudio, pues Turbo y Apartadó limitan sus análisis con la identificación de impactos atmosféricos por agroquímicos; además, las concentraciones de clorotalonil se estudian aisladas de otros productos que se emplean en la región simultáneamente. Los parámetros medidos en la ciudad de Quibdó no son suficientes para conocer la calidad del aire de
Tabla 2

Porcentajes de remoción industrias aceite de palma

\begin{tabular}{|c|c|c|c|c|}
\hline \multirow[t]{2}{*}{ Empresas } & \multicolumn{3}{|c|}{ Parámetro } & \multirow[b]{2}{*}{ DQO } \\
\hline & $\begin{array}{c}\text { Grasas y } \\
\text { aceites }\end{array}$ & SST & $\mathrm{DBO}_{5}$ & \\
\hline $\begin{array}{l}\text { Valor permisible DEC } \\
1594 / 84 \%\end{array}$ & 80,00 & 80,00 & 80,00 & 80,00 \\
\hline Astorga \% de remoción & 96,27 & 97,76 & 99,14 & 98,20 \\
\hline Araki \% remoción & 99,12 & 89,06 & 99,69 & 99,17 \\
\hline Palmeiras \% remoción & 99,6 & 98,80 & 97,86 & 99,46 \\
\hline
\end{tabular}

este centro poblado; si bien la Resolución 005 de 1997 reglamenta los niveles permisibles de emisión de contaminantes producidos por fuentes móviles terrestres, no se incluyen valores permisibles de gases contaminantes por motocicletas, vehículos a los cuales es necesario prestar especial atención, sobre todo en ciudades como Quibdó y Turbo donde el rapimoterismo (mototaxis) es una fuente importante de empleo. En Buenaventura, aunque se miden los gases considerados los principales contaminantes emitidos por fuentes móviles y fijas, el estudio se desarrolló hace más de siete años, lo que amerita el desarrollo de monitoreos que precisen las condiciones actuales de la calidad del aire. Las empresas procesadoras de palma de aceite de la ciudad de Tumaco generan información tanto de emisiones atmosféricas como de vertimientos líquidos; sin embargo, limitan sus análisis sólo a las chi- 


\section{Bioetnia Volumen 5 No 1 (enero-junio), 2008}

meneas del gremio aceitero, lo cual sugiere un sesgo porque no se contemplan otras fuentes de emisión, ni las concentraciones de contaminantes en el ambiente. Para generar datos más precisos y que permitan tener una versión definitiva de los efectos de la calidad del aire sobre la salud de los habitantes de los centros de mayor poblamiento de la región, es necesario medir las concentraciones de partículas sólidas totales (PST) en las diferentes ciudades de muestreo, teniendo en cuenta los niveles de infecciones agudas que se dan en pacientes de zonas aledañas a centros poblados que no son objeto de estudio.

Por otra parte, las principales fuentes de contaminación acuática en la región se pueden clasificar como urbanas y agroindustriales; ambas se conjugan con el desarrollo de algunos centros poblados y se hacen críticas al presentarse densidades poblacionales que corresponden a 832.924 habitantes distribuidos mayoritariamente en Buenaventura (37\%), seguido de Apartadó, Tumaco y Turbo (16\% cada uno). Además, los deficientes procesos de saneamiento derivados de la generación de desechos sólidos en asentamientos urbanos y vertimiento directo de aguas servidas a las corrientes de agua, repercuten en la calidad ambiental, porque los sistemas de tratamiento y las redes de acueducto y alcantarillado son insuficientes en unos casos, deficientes y ausentes en otros. Los tipos de contaminantes y efectos más sobresalientes se expresan en el Urabá (Apartadó y Turbo), principalmente por su importancia como zona bananera; y en Buenaventura por la descarga de metales pesados provenientes de zonas mineras en el río Dagua y al vertimiento de los derivados del petróleo. Sin embargo, la mayor parte de estudios de calidad del agua en la región, se reducen a los factores físicoquímicos dejando despejados los temas relacionados con estructura trófica y composición de las comunidades sensibles a efectos de contaminación por lo cual se hace necesaria la investigación en ecosistemas dulceacuícolas, marinos y costeros con miras a la determinación y evaluación de indicadores biológicos de sistemas alternos de tratamiento de aguas residuales, los cuales a su vez, deberán complementarse con el seguimiento de las condiciones fisicoquímicas y microbiológicas.

Adicionalmente la información suministrada por los hospitales se refiere a generalización de las infecciones e inadecuado diligenciamiento de las fichas epidemiológicas, lo cual se debe en parte a las limitaciones logísticas para el análisis especializado de agentes tóxicos en el organismo, identificados en el ambiente como es el caso de metales pesados, que se presume es un motivo de consulta recurrente.

Finalmente, es importante generar un nivel de acercamiento a las condiciones medio ambientales de estos centros poblados con el fin de establecer el escenario actual de los recursos naturales y determinar cuánto cambian a futuro con la intervención de nuevos proyectos de desarrollo. En este sentido, los procesos de investigación deben ser articulados, permitiendo hacer análisis a nivel regional y no sectorial; para ello se requiere una labor conjunta de las CARs, instituciones y municipalidades.

\section{AGRADECIMIENTOS}

Los autores agradecen a las Corporaciones Autónomas Regionales del Chocó Biogeográfico (CORPOURABÁ, CODECHOCÓ, CVC, CORPONARIÑO), a la Universidad Tecnológica del Chocó, Universidad de Antioquia Sede Urabá, Universidad del Valle, a la Alcaldía de Buenaventura y al Hospital Regional Departamental de Buenaventura por la información suministrada para el desarrollo de este trabajo.

\section{LITERATURACITADA}

Álvarez, Vy M. Cuesta. 2005. Evaluación ambiental del sector de la parte alta de la quebrada el Caraño municipio de QuibdóChocó. Trabajo de grado para optar el título de Biólogos con énfasis en Recursos Naturales. Universidad Tecnológica del Chocó, Quibdó, Colombia.

Análisis Ambiental S.A. 2003, 2005, 2006 y 2007. Informe caracterización de vertimientos sistema de tratamiento de residuos líquidos industriales. San Andrés de Tumaco, Nariño, Colombia.

Andrade F. y E. Córdoba. 2004 Incidencia de la emisión de gases de los vehículos de servicio público sobre el aire de la ciudad de Quibdó, Chocó. Trabajo de grado para optar el título de Ingenieros Ambientales. Universidad Tecnológica del Chocó, Quibdó, Colombia.

AUGURA y CORPOURABÁ. 2000. Plan de manejo ambientalparael manejo integral de agroquímicos en la zona de Urabá. Medellin: Universidad de Antioquia, Facultad Nacional de Salud Pública.

Bejarano P. y E. Palacios. 2004. Diversidad y ecología del orden trichoptera en la quebrada la Francisca en el Municipio de Quibdó, Chocó. Trabajo de grado para optar el título de Biólogos con énfasis en Recursos Naturales, Universidad Tecnológica del Chocó, Quibdó, Colombia.

Caicedo W., F. Mena. 2005. Caracterización fisicoquimica microbiológica de la quebrada el Caraño y determinación de las 


\section{Calidad del aire y el agua en el Chocó Biogeográfico colombiano. Y Torres, T López, F Carabalí}

condiciones socioeconómicas de la población del área de influencia directa. Trabajo de grado para optar el título de Biólogos con énfasis en Recursos Naturales, Universidad Tecnológica del Chocó, Quibdó, Colombia.

Castro C. y Y. Torres. 2004. Caracterización limnológica de la parte media alta del río Atrato en peróodo de agosto altos y bajos, sector barrio calle Quibdó, Chocó. Trabajo de grado para optar el título de Biólogos con énfasis en Recursos Naturales. Universidad Tecnológica del Chocó, Quibdó, Colombia.

CODECHOCÓ. 2000. Plan degestión ambiental regionaldeldepartamento del Chocó 2001-2010. Quibdó, Chocó.

Cuesta I. y J. Reales. 2007. Monitoreo de las emisiones de gases producidas porlas motocicletas en la ciudad de Quibdó, Chocó, Colombia. Trabajo de grado para optar el título de Ingenieros Ambientales, Universidad Tecnológica del Chocó, Quibdó, Colombia.

ESRI. Environmental Systems Research Intitute. 2000. ArcView GIS versión 3.2 Manual de referencia (en línea): Lugar ESRI (on-line) http://www.esri.com/

García J. y J. Correa. 2006. Macroinvertebrados y peces asociados a las raices sumergidas de mangle rojo en las bahías de Turbo y ElUno, Golfo de Urabá (Caribe colombiano). Trabajo de Grado para optar el título de Ecólogos de Zonas Costeras, Universidad de Antioquia, Sede Urabá. Programa de Ciencias del Mar, Turbo, Antioquia.

Gaviria D. 2006. Macroinvertebrados asociados a macroalgas marinas de los litorales rocosos del golfo de Urabá. Trabajo de grado para optar el título de Ecóloga de Zonas Costeras. Universidad de Antioquia, Sede Urabá, Programa de Ciencias del Mar, Turbo, Antioquia.

Golden Software Inc. Surface Mapping Sistem, Surfer, Versión 8.02 (on-line) http: / /www.goldensoftware. com 1993-2002.

Hinestroza L. 2002. Selección de tecnología para el tratamiento de las aguas residuales provenientes de la industria pesquera en el sectorde Pueblo Nuevo, Buenaventura (Pesquera Dispemar). Tesis de Grado. Programa académico de Ingeniería Sanitaria. Universidad del Valle, Valle del Cauca, Colombia.

INVEMAR. 2002. Informe del estado de los ambientes marinos y costeros en Colombia: año 2001. Serie de publicaciones periódicas. Santa Marta, Colombia.

INVEMAR. 2004. Informe delestado de los ambientales marinos y costeros en Colombia. Región Pacífico continental. Santa Marta, Colombia.

Lozano, Y. 2006. Dinámica de la acumulación de Cu, Pby Hg en dos especies de algas bénticas y su uso como bioindicadores en la bahía de Buenaventura, Pacífico colombiano. Trabajo de grado para optar el título de Biólogo. Universidad del Valle, Facultad de Ciencias, Cali, Colombia.

Lozano, J. 1998. Evaluación de la contaminación por hidrocarburos en la bahía de Buenaventura y Málaga. Programa de Química, Universidad del Valle, Valle del Cauca, Colombia.

Marín, D y Moreno E. 2005. Caracterización fisico-quimicamicrobiológica de la quebrada la Aurora y determinación de las condiciones socioeconómicas de la población del área de influencia directa. Trabajo de grado para optar el título de Biólogos con énfasis en Recursos Naturales. Universidad Tecnológica del Chocó, Quibdó, Colombia.

Ministerio de Salud. Decreto 475 de 1998. Norma técnica de calidad delagua potable. Colombia.

Ministerio de Salud. Decreto 1594 1984. Norma técnica de usos del agua y residuos líquidos. Colombia.

Ministerio de Salud. Decreto 02 de 1982. Reglamentación parcial del Titulo I de la Ley 09 de 1979 y el Decreto Ley 2811 de 1974 en cuanto a emisiones atmosféricas. Colombia.

Ministerio de Medio Ambiente. Decreto 948 de 1995. Reglamentación parcial de la Ley 23 de 1973, los artículos 33, 73, 74, 75 y 76 del Decreto-Ley 2811 de 1974; los artículos 41, 42, 43, 44, 45, 48 y 49 de la Ley 9 de 1979; y la Ley 99 de 1993, en relación con la prevención y control de la contaminación atmosférica y la protección de la calidad del aire. Colombia

Ministerio de Medio Ambiente. Resolución 005 de 1997. Norma técnica de niveles de emisión de fuentes móviles. Colombia

Ministerio de Protección Social, Ministerio de Vivienda y Desarro1lo Territorial. Decreto 1575 de 2007. Sistema para la protección y control de la calidad del agua para consumo humano. Colombia.

Ministerio de Transporte. 2005. Infraestructura de transporte para la competitividad del país. República de Colombia (online). http:// www.mintransporte.gov.co

Municipio de Buenaventura. 2000. Plan de ordenamiento territorial de Buenaventura. Departamento de Planeación Municipal, Valle del Cauca, Colombia.

Municipio de Guapi. 2000. Plan de ordenamiento territorial de Guapi. Departamento de Planeación Municipal, Cauca, Colombia.

Muñoz, A., C. Quirozy J. Paz. 2007. Efectos de la contaminación atmosférica sobre la salud de adultos que laboran a diferentes niveles de exposición. Universidad de Antioquia. Rev Fac Nac Salud Publica. 25: 2: 85-94.

Ortega, L. 1996. Determinación de los niveles de plomo y mercurio en el agua de mar de la bahía de Buenaventura. Trabajo de grado para optar el título de Químico. Universidad Santiago de Cali. Departamento de Química, Cali, Colombia.

Palacios, A. y A. Romaña. 2004. Caracterización limnológica en las aguas altas y bajas en la parte media alta del río Atrato en el sector comprendido entre el hospital San Fco de Asís, hasta la desembocadura de la quebrada el Caraño, Quibdó, Chocó. Trabajo de grado para optar el título de Biólogos con énfasis en Recursos Naturales, Universidad Tecnológica del Chocó. Quibdó, Colombia.

Palacios, J. y J. Pino. 2005. Caracterización y estudio de la calidad delagua en la micro cuenca el Caraño, Quibdó, Chocó. Trabajo de grado para optar el título de Biólogos con énfasis en Recursos Naturales. Universidad Tecnológica del Chocó, Quibdó, Colombia.

Rosero, M. 2000. Monitoreo de calidad de aire en Buenaventura unidad móvil periodo abril-agosto. CVC. Valle del Cauca, Colombia.

Roqueme, E. y D. Blanco. 2005. Evaluación de plaguicidas organoclorados en sedimentos en la parte baja del río Turbo. Trabajo de grado para optar el título de Ecólogo de Zonas Costeras, Universidad de Antioquia, Sede Urabá. Programa de Ciencias del Mar, Turbo, Antioquia.

Tovar, J. 1995. Efectos de la polución por aguas residuales domésticas sobre comunidades macrobentonicas en playas lodosas intermareales en la Bahía de Buenaventura. Programa de Biología. Universidad del Valle, Valle del Cauca, Colombia.

Unidad Administrativa Especial de la Aeronáutica Civil (UAEAC). 2006. Informe monitoreo ambiental de agua y aire. San Andrés de Tumaco, Nariño, Colombia. 